\title{
LYAPUNOV EXPONENTS FOR A STOCHASTIC ANALOGUE OF THE GEODESIC FLOW
}

\author{
A. P. CARVERHILL AND K. D. ELWORTHY
}

\begin{abstract}
New invariants for a Riemannian manifold are defined as Lyapunov exponents of a stochastic analogue of the geodesic flow. A lower bound is given reminiscent of corresponding results for the geodesic flow, and an upper bound is given for surfaces of positive curvature. For surfaces of constant negative curvature a direct method via the Doob $h$-transform is used to determine the full Lyapunov structure relating the stable manifolds to the horocycles.
\end{abstract}

1. Introduction. In [3, Theorem 2.1] Carverhill defines the Lyapunov spectrum and filtration for the flow of a smooth stochastic dynamical system (SDS) on a smooth compact Riemannian manifold (see Theorem 1 below). This is a generalisation of the work of Ruelle [25] (see also [21, 24]) for a deterministic system. See [10] (also [6]) for a detailed discussion of stochastic systems and their flows, or [1] for an introductory article. Associated to a smooth Riemannian manifold $M$ is an SDS on the orthonormal frame boundle $O M$, called the Canonical SDS for $M$, and which is described in Elworthy [10, Chapter 7, Example 1A(iii), p. 112] (see also references on p. 157 of $[\mathbf{1 0}]$, and Sunada [27]). It seems that the Canonical SDS is the simplest stochastic system which can be 'canonically' defined given the Riemannian structure, i.e. without an arbitrary choice of additional structure on $M$. Our aim in this paper is to study the Lyapunov spectrum and filtration for the Canonical SDS.

Since our system is canonical in the sense above, it follows that the spectrum and filtration are determined by the Riemannian structure on $M$. In Theorem 1 below we state Theorem 2.1 of $[3]$ as it applies to the Canonical SDS.

We will denote the Canonical SDS by $(X, B)$, and its flow by $\xi_{t}(\omega)$. Thus, $B$ is a Brownian motion on $\mathbf{R}^{n}(n=\operatorname{dim} M)$, and $X$ is a bundle morphism $O M \times$ $\mathbf{R}^{n} \rightarrow T O M$, defined such that for any frame $u \in O_{X} M$ (i.e. a linear isometry $\left.\mathbf{R}^{n} \rightarrow T_{x} M\right)$ and any $e \in \mathbf{R}^{n}, X(\mathfrak{u}) e$ is the horizontal lift to $T O M$ of $\mathfrak{u}(e) \in T_{x} M$, with respect to the Riemannian connection on $O M$ (see [10, Appendix B]). Eells and Elworthy (see [10, Theorem 12B, p. 159]) showed that the solutions to $(X, B)$ project to heat flow on $M$, i.e. the transition probabilities $p_{t}(x, d y)$ on $M$ given by $p_{t}(x, B)=P\left\{\omega: \pi\left(\xi_{t}(\omega) \mathfrak{u}\right) \in B\right\}$ are the fundamental solutions to the heat equation on $M$. (Here, $B$ is any Borel set in $M, \pi$ is the bundle projection $O M \rightarrow M$, and $\mathfrak{u}$ is any frame at $x$ - the choice does not affect $p_{t}(x, B)$.)

Received by the editors March 27, 1985. The principal results given here were presented to the VII Escuela Latinoamericana de Matemáticas, USB-Caracas, July 16-27, 1984.

1980 Mathematics Subject Classification (1985 Revision). Primary 58G32, 58F17; Secondary $58 \mathrm{~F} 11,53 \mathrm{C} 20,60 \mathrm{H} 10$.

Key words and phrases. Lyapunov exponents, geodesic flow, stochastic differential equations, Brownian motion, Riemannian manifolds, hyperbolic space, horocycles. 
The following theorem gives the definition and existence of the Lyapunov spectrum and filtration for the Canonical SDS.

THEOREM 1. Take any invariant measure $\nu$ for the Markov process on $O M$ associated with $\xi_{t}(\omega)$. (Transition probabilities $q_{t}(\mathfrak{u}, B)=P\left\{\omega: \xi_{t}(\omega) \mathfrak{u} \in B\right\}, B$ Borel in $O M$.) Then there exists a set $\Gamma \subset O M \times \Omega$ of full $\nu \otimes P$-measure such that for each $(\mathfrak{u}, \omega) \in \Gamma$ we have a Lyapunov spectrum

$$
\lambda_{\mathfrak{u}}^{(1)}<\lambda_{\mathfrak{u}}^{(2)}<\cdots<\lambda_{\mathfrak{u}}^{(r)}
$$

and associated filtration

$$
\{0\} \equiv V_{(\mathfrak{u}, \omega)}^{(0)}<V_{(\mathfrak{u}, \omega)}^{(1)}<\cdots<V_{(\mathfrak{u}, \omega)}^{(r)} \equiv T_{\mathfrak{u}} O M .
$$

Thus, if $\mathfrak{y} \in T_{\mathfrak{u}} O M, \mathfrak{y} \in V_{(\mathfrak{u}, \omega)}^{(i)} \backslash V_{(\mathfrak{u}, \omega)}^{(i-1)}(i=1, \ldots, r)$, then

$$
\frac{1}{t} \log \left\|T \xi_{t}(\omega) \mathfrak{y}\right\| \rightarrow \lambda_{\mathfrak{u}}^{(i)} \quad \text { as } t \rightarrow \infty \text {. }
$$

Given $\mathfrak{u}$, the dimensions of the subspaces in the filtration are independent of $\omega$ for $(\mathfrak{u}, \omega) \in \Gamma$. We define the multiplicity of $\lambda_{\mathfrak{u}}^{(i)}$ to be $\operatorname{dim} V_{(\mathfrak{u}, \omega)}^{(i)}-\operatorname{dim} V_{(\mathfrak{u}, \omega)}^{(i-1)}$ for such $\omega$.

ProOF. Note that $M$ being compact implies that $O M$ is compact; therefore this result follows from [3, Theorem 2.1], except that as we have stated that Theorem, the spectrum can depend on $\omega \in \Omega$. However, if $\nu$ is ergodic, then the spectrum (and its multiplicities) are independent of $\mathfrak{u}$ and $\omega$, and since any invariant measure is a combination of ergodic measure, which are supported on disjoint (though perhaps not closed) sets (see Yosida [28, Chapter 13, §4], also [3, §1.2]), we can deduce that the spectrum above and its multiplicities (and those of [3, Theorem 2.1]) are independent of $\omega \in \Omega$ if $\nu$ is merely invariant.

The statement of Theorem 1 used a Riemannian metric on $O M$. Since $O M$ is compact for $M$ compact the choice of metric was unimportant. However we will often use the natural metric, or Sasaki metric, of $O M$. This is defined using the connection on $O M$ by requiring that for each $\mathfrak{u}$ of $O M$ the horizontal and vertical tangent subspaces of $\mathfrak{u}$ are orthogonal, that $T_{\mathfrak{u}} \pi$ is isometric on the horizontal subspace, and that the connection form maps the vertical subspace at $\mathfrak{u}$ isometrically onto the lie algebra $\mathfrak{o}(n)$ with its usual (Hilbert-Schmidt) metric. This metric is clearly invariant under the action of isometries of $M$ and the right action of $O(n)$, as is the corresponding measure: the Liouville measure (or Liouville volume).

The construction of the Canonical SDS is analogous to that of the ordinary dynamical system (vector field) $A$ on $T M$ (or the sphere bundle $S M$ ), whose flow is the geodesic flow: $A$ is defined such that for each $v \in T M$ (or $S M$ ), $A(v)$ is the horizontal lift to TTM (or TSM) of $v$ with respect to the Riemannian connection on $T M$. Thus, our aim is analogous to the study of the Lyapunov spectrum for the geodesic flow on $T M$ (or $S M$ ) for which see $[\mathbf{1}, \mathbf{2 7}]$.

It will be seen that many of our techniques and results are also analogous. For instance in $\S 2$ we show that the flow of $(X, B)$ preserves the Liouville measure on $O M$, and the Lyapunov exponents sum to zero. Also in $\S 5$ we show that we can give a full description of the Lyapunov spectrum and filtration when $M$ is the hyperbolic 2 -space. Note however that our approach to the question of ergodicity of the flow of $(X, B)$ (see $\S 3)$ is different from the approach of [1] for the geodesic flow. 
The following description of $(X, B)$ and its flow, in terms of the connection on $T M$, will be useful in the sequel (see Elworthy [10, Chapter 7, §11], also [27]):

Denote by $\left\{e_{1}, \ldots, e_{n}\right\}$ the standard basis in $\mathbf{R}^{n}$. We will sometimes express an orthonormal frame $\mathfrak{u}: \mathbf{R}^{n} \rightarrow T_{x} M$ as $\left(u_{1}, \ldots, u_{n}\right)$, where $u_{i}=\mathfrak{u}\left(e_{i}\right)$, which is an orthonormal base in $T_{x} M$. Denote the vector field $X(-) e_{i}: O M \rightarrow T O M$ by $X^{i}$, and its flow by $\xi_{t}^{i}$. Then the solution $\xi_{t}^{i}(\mathfrak{u})$ is the parallel translation of $\left(u_{1}, \ldots, u_{n}\right)$ along the geodesic starting from $u_{i}$. This description of $\xi_{t}^{i}$ characterises $X^{i}$ completely, and hence $(X, B)$ itself, and it follows that for any $e \in \mathbf{R}^{n}, X(-) e$ is the vector field whose solution starting from a frame $u$ is the parallel translation of $\left(u_{1}, \ldots, u_{n}\right)$ along the geodesic starting in the direction of $u(e)$.

Parallel translation of a frame $\mathfrak{u} \in O_{x} M$ along a geodesic starting in the direction of $v \in T_{x} M$ can be interpreted as rolling the manifold without slipping along a straight line in $\mathbf{R}^{n}$ : we start with $x$ sitting at the origin in $\mathbf{R}^{n}$, and lined up according to $\mathfrak{u}$, i.e. with $e_{i} \in \mathbf{R}^{n}$ against $u_{i} \in T_{x} M$, and we roll along the line in $\mathbf{R}^{n}$ which is lined up against $v$. At each time $t$ the parallel translation $\left(u_{1}^{t}, \ldots, u_{n}^{t}\right)$ of the frame is lined up against the frame in $\mathbf{R}^{n}$ which is parallel to the standard basis at the origin. Now, take a piecewise linear approximation $\left(X, B^{\Pi}\right)$ to $(X, B)$, where $\Pi=\left(0, t_{1}, t_{2}, \ldots\right)$, so that for each $\omega \in \Omega, B_{t_{i}}^{\Pi}(\omega)=B_{t_{i}}(\omega)$ and $B_{t}^{\Pi}(\omega)$ is linear between $0, t_{1}, t_{2}, \ldots$ (see $\left.[\mathbf{1 0}, \mathbf{6}]\right)$. Then for each $\omega$ the solution to $\left(X, B^{\Pi}\right)$ starting from $\mathfrak{u} \in O_{x} M$ is obtained by rolling $M$ without slipping along the path $B_{t}^{\Pi}(\omega)$ in $\mathbf{R}^{n}$, starting with $x$ sitting at the origin, and lined up according to $\mathfrak{u}$. (This is the Cartan development.) The flow of $(X, B)$ itself can be obtained from that of $\left(X, B^{\Pi}\right)$ by letting mesh $(\Pi)$ tend to zero. (n.b. We can only define the Cartan development along a piecewise differentiable path. This is why we must go via the piecewise linear approximation here.)

\section{Preliminary results.}

PROPOSITION 2.1. $(X, B)$ is invariant under isometries of $M$, i.e. if $h: M \rightarrow$ $M$ is an isometry, then for each $\mathfrak{u} \in O M, e \in \mathbf{R}^{n}$ we have

$$
\operatorname{TOhX}(\mathfrak{u}) e=X(O h(\mathfrak{u})) e
$$

where $O h: O M \rightarrow O M$ is given by $O h(\mathfrak{u}) e_{i}=T h\left(\mathfrak{u}\left(e_{i}\right)\right)(i=1, \ldots, n)$.

PROOF. The proof is immediate from the fact that $(X, B)$ is canonically defined with respect to the Riemannian metric.

For the following we should note that our requirement for a flow of an S.D.E. is that it be continuous in the space variable (almost sure smoothness will then follow automatically).

Proposition 2.2. Denote by $I_{s}(M)$ the compact Lie group of isometries of $M$. Then for a.e. $\omega$ the flow is invariant under the action of $I_{s}(M)$, i.e. for a.e. $\omega$ we have for all $h \in I_{s}(M), t>0, \mathfrak{u} \in O M$ that

$$
\xi_{t}(\omega)(O h(\mathfrak{u}))=O h\left(\xi_{t}(\omega) \mathfrak{u}\right)
$$

PROOF. This follows from the following general lemma.

LEMMA 2.3. Suppose the smooth $S D S(Y, z)$ on the smooth compact manifold $N$ is invariant under the action of a compact Lie group $H$ of smooth diffeomorphisms on $N$, i.e. for all $h \in H, x \in N, e \in \mathbf{R}^{n}$ we have

$$
Y(h x) e=T h \cdot Y(x) e .
$$


Then for a.e. $\omega \in \Omega$, the flow $\zeta_{t}(\omega)$ is invariant under the action of $H$, i.e. for all $x \in N, h \in H, t>0$ we have

$$
h\left(\zeta_{t}(\omega) x\right)=\zeta_{t}(\omega)(h x) .
$$

ProOF. Define an SDS $(\tilde{Y}, z)$ on the compact manifold $N \times H$ by $\tilde{Y}(x, h) e=$ $(Y(x) e, 0)$, and denote its flow by $\tilde{\zeta}_{t}(\omega)$. From [6] we see that $\tilde{\zeta}_{t}(\omega)$ is a.s. uniquely determined by the condition that for each $(x, h) \in N \times H, \zeta_{t}(\omega)(x, h)$ is the solution to $(\tilde{Y}, z)$ starting from $(x, h)$, and tht $\tilde{\zeta}_{t}(\omega)$ is a.s. continuous in $(x, h)$. Also, it is clear that $(x, h) \mapsto\left(\zeta_{t}(\omega) x, h\right)$ is a flow of $(\tilde{Y}, z)$. Now, transforming the equation for $\zeta_{t}(\omega) x$ via $h$ and using the Itô formula, we can deduce that

$$
d\left(h_{\zeta_{t}}(\omega) h^{-1} x\right)=Y\left(h_{\zeta_{t}}(\omega) h^{-1} x\right) \circ d z_{t},
$$

i.e. for each $h \in H, h_{\zeta_{t}}(\omega) h^{-1}$ is a version of the flow of $(Y, z)$. Since this is continuous in $h$, we can also deduce that $(x, h) \mapsto\left(h_{\zeta_{t}}(\omega) h^{-1} x, h\right)$ is a version of the flow of $(\tilde{Y}, z)$, and the result follows by a.s. uniqueness of this flow.

Proposition 2.4. (i) For a.e. $\omega$ the flow $\xi_{t}(\omega)$ preserves the (normalised) Liouville volume $\mu$ on $O M$.

(ii) The Markov process on $O M$ associated with $\xi_{t}(\omega)$ has $\mu$ as an invariant measure.

ProOF. (i) See [20]. The result follows from the fact that with respect to the Riemannian metric on $O M$, we have $\operatorname{div} X^{i}=0$, which implies that the flow $\xi_{t}^{i}$ preserves the Liouville volume. $\left(X^{i}, \xi_{t}^{i}\right.$-notation of $\S 1$.)

(ii) Elementary from part (i).

Proposition 2.5. The sum of the Lyapunov spectral elements (with multiplicities) is zero for the Canonical SDS.

PROOF. In view of Proposition 2.4(i), this follows from the general result:

PROPOSITION 2.6. Consider the smooth stochastic flow $\zeta_{t}(\omega)$ on the smooth compact Riemannian manifold $N$.

(i) If the Lyapunov spectrum exists at $(x, \omega)$, then the sum of the Lyapunov spectral elements (with multiplicities) at $(x, \omega)$ is given by

$$
\lim _{t \rightarrow \infty} \frac{1}{t} \log \operatorname{det}\left[\left.T_{\zeta_{t}}(\omega)\right|_{T_{x} N}\right] \text {. }
$$

(Here by "det" we mean the determinant of the linear map $\left.T_{\zeta_{t}}(\omega)\right|_{T_{x} N}: T_{x} N \rightarrow$ $T_{\zeta_{t}(\omega) x} N$, with respect to the Riemannian inner product on these tangent spaces.)

(ii) If there is a smooth measure on $N$ whose density $v$ is never zero, and for a.e. $\omega$ the flow $\zeta_{t}(\omega)$ preserves this measure, then the sum of the exponents is zero.

PROOF. (i) In the proof of the existence of the Lyapunov spectrum [3, Theorem 2.1], we embed $N$ in a Euclidean space, and work with the Riemannian metric on $N$ induced from the embedding. From the proof we see that if the spectrum exists at $(x, \omega)$, then the sum of the spectral elements here is

$$
\lim _{t \rightarrow \infty} \frac{1}{t} \log \operatorname{det}^{E}\left[T_{\zeta_{t}}(\omega) \mid T_{x} N\right]
$$


where $\operatorname{det}^{E}$ is the determinant taken with respect to the inner products on the tangent spaces, induced from the embedding. The result follows because the inner products on the tangent spaces are uniformly equivalent.

(ii) Dividing the Riemannian inner product on each $T_{x} N(x \in N)$ by $v(x)^{2}$ yields an equivalent Riemannian metric in which for a.e. $\omega, \operatorname{det}\left[\left.T_{\zeta_{t}}(\omega)\right|_{T_{x} N}\right]=1$ for all $x$, all $t \geq 0$. Therefore the result follows from part (i).

3. Constancy of the Lyapunov spectrum. If $\operatorname{dim} M \equiv n>1$, then the Canonical SDS is degenerate $(\operatorname{dim}$ noise $\equiv n<\operatorname{dim} O M \equiv n+(n-1) n)$, and we do not know a priori whether the Markov process associated with it is ergodic, with respect to the measure $\mu$ (i.e. the Liouville volume) on $O M$. In fact the Canonical SDS on the flat torus is an example which is not ergodic: this is clear from the fact that parallel translation of a frame cannot alter its angle of inclination. Thus, we cannot deduce from [3] that the spectrum of Theorem 1 above is independent of $\mathfrak{u} \in O M$. Our aim in this section is to show that this is nevertheless true. The result follows essentially because the system is invariant in the sense of Lemma 3.1 below, with respect to rotations of the frame.

LEMMA 3.1. Take any $g \in O(n)$.

(i) Then for all $t>0, \mathfrak{u} \in O M$, a.e. $\omega \in \Omega$, we have

$$
\left[\xi_{t}(\omega) \mathfrak{u}\right] \circ g=\xi_{t}\left(g^{-1} \omega\right)(\mathfrak{u} \circ g) .
$$

(ii) For all $t>0, \mathfrak{y} \in T O M$, a.e. $\omega \in \Omega$, we have

$$
\left[T \xi_{t}(\omega) \mathfrak{y}\right] \tilde{g}=T \xi_{t}\left(g^{-1} \omega\right)(\mathfrak{y} \tilde{g}) .
$$

(Here, $\tilde{g}$ is the map TOM $\rightarrow$ TOM (written on the right: $\mathfrak{y} \rightarrow \mathfrak{y} \tilde{g}$ ) defined by $\mathfrak{y} \tilde{g}=d(\mathfrak{u} \circ g) / d t$ if $\mathfrak{u}_{t}$ is a curve on $O M$, and $\mathfrak{y}=d \mathfrak{u}_{t} / d t$.)

The map $\tilde{g}$ restricts to a linear isometry $T_{\mathfrak{u}} O M \rightarrow T_{\mathfrak{u} \circ g} O M$ for each $\mathfrak{u} \in M$.

ProOF. (i) This is easily true if we take a piecewise linear approximation to the system (see [6, Proposition 4.1]). To obtain the result stated use the fact that the piecewise linear approximation converges in measure uniformly over $\mathfrak{u} \in O M$, $t \in[O, T]$ (any $T>0$ ), and the fact that the map $\omega \mapsto g \omega$ preserves the measure $P$ on $\Omega$.

(ii) Differentiate the equation of part (i), and regarding $g$ as a map $O M \rightarrow O M$, $\mathfrak{u} \mapsto \mathfrak{y} \circ g$, take $\tilde{g}$ to be its derivative.

LEMMA 3.2. For each $q=1, \ldots, n$, put

$$
f^{q}(\mathfrak{u}, \omega)=\lim _{t \rightarrow \infty} \frac{1}{t} \log \left\|\left[T \xi_{t}(\omega)_{\mathfrak{u}}\right]^{\Lambda_{q}}\right\|,
$$

whenever this limit exists. Here, by $T \xi_{t}(\omega)_{\mathfrak{u}}$ we mean the linear map

$$
\left.T \xi_{t}(\omega)\right|_{T_{u} O M}: T_{u} O M \rightarrow T_{\xi_{t}(\omega) \mathfrak{u}} O M,
$$

and by $\Lambda_{q}$ we mean the qth exterior power. Take any invariant measure $\nu$ for the Markov process on $O M$ associated with $\xi_{t}(\omega)$. Then for $\nu$-a.e. $u$, and given $\mathfrak{u}$, for a.e. $\omega$, we can define $f^{q}(\mathfrak{u}, \omega)$, and it is a.s.-independent of $\omega$. For such $\mathfrak{u}$ denote by $\bar{f}^{q}(\mathfrak{u})$ the a.s. value of $f^{q}(\mathfrak{u}, \omega)$. Then $\bar{f}^{q}(\mathfrak{v})$ exists and is equal to $\bar{f}^{q}(\mathfrak{u})$ for all $\mathfrak{v} \in O_{\pi(u)} M$. 
ProOF. The fact that $\bar{f}^{q}(\mathfrak{u}, \omega)$ can be defined for $(\mathfrak{u}, \omega)$ as in the statement can be deduced using the ideas of the proof of Theorem 2.1 in [3]. In fact from that proof it follows that $f^{q}(\mathfrak{u}, \omega)$ is $\nu \otimes P$-a.s. invariant under the time shift

$$
\Phi_{T}: O M \times \Omega \rightarrow O M \times \Omega ; \quad(\mathfrak{u}, \omega) \rightarrow\left(\xi_{T}(\omega) \mathfrak{u}, \Theta_{T}(\omega)\right)
$$

(any $T>0$ ). Now, if the Markov process on $O M$ is ergodic with respect to $\nu$ (restricting attention to time increments of length $T$ ), then by [3, Appendix B, Theorem B], this time shift is ergodic with respect to $\nu \otimes P$, and so $f^{q}(u, \omega)$ is $\nu \otimes P$ a.s. constant. To see that for $\nu$ merely invariant we have $f^{q}(\mathfrak{u}, \omega)$ a.s. independent of $\omega$ for a.s. $\mathfrak{u}$, consider the decomposition given by Yosida [28, Chapter 13, §4] of $M$ into uniquely ergodic components (see [3, $\S 1.2]$, also the proof of Theorem 1 above): $f^{q}(\mathfrak{u}, \omega)$ is a.s. independent of $\omega$ on each of these components, and they make up a set of $\nu$-full measure if $\nu$ is any invariant measure.

We now show that if $\mathfrak{v} \in O_{\pi(\mathfrak{u})} M$, then $\bar{f}^{q}(\mathfrak{v})=\bar{f}^{q}(\mathfrak{u})$. Take $g \in O(n)$ such that $\mathfrak{u} \circ g=\mathfrak{v}$. Since $\tilde{g}$ is an isometry $T_{\mathfrak{w}} O M \rightarrow T_{\mathfrak{w} \circ g} O M$ (any $w \in O M$ ), we have

$$
\left\|\left[T \xi_{t}(\omega)_{\mathfrak{u}}(-)\right]^{\Lambda_{q}}\right\|=\left\|\left[\left[T \xi_{t}(\omega)_{\mathfrak{u}}(-)\right] \tilde{g}\right]^{\Lambda_{q}}\right\| .
$$

Therefore (using Lemma 3.1(ii)) we have $f^{q}(\mathfrak{u}, \omega)=f^{q}\left(\mathfrak{u} g, g^{-1} \omega\right)$ for a.e. $\omega$, and hence (using the fact that $g: \Omega \rightarrow \Omega$ preserves the measure $P$ ) we have $\bar{f}^{q}(\mathfrak{u})=$ $\bar{f}^{q}(\mathfrak{v})$.

THEOREM 3.3. If $\nu$ is any invariant measure for the Markov process on $O M$ associated with $\xi_{t}(\omega)$, then the Lyapunov spectrum for $\xi_{t}(\omega)$ and its multiplicities are $\nu \otimes P$-a.s. constant on $O M \times \Omega$.

ProOF. For each $(u, \omega)$ for which the functions $f^{q}$ are defined $(q=1, \ldots, n)$, the Lyapunov spectrum at $(\mathfrak{u}, \omega)$ and its multiplicities can be constructed from the values of these functions at $(\mathfrak{u}, \omega)$ (see Ruelle [25, Proposition 1.3]). Also, from the invariance of $\nu$ on $O M$ we can deduce that of $\pi(\nu)$ on $M$ for the Markov process associated with heat flow, and in fact we must have $\pi(\nu)=\rho$ (Riemannian volume) because this Markov process is uniquely ergodic. Using this and Lemma 3.2 we see that for $\rho$-a.s. $x \in M, \bar{f}^{q}$ is constant for $\mathfrak{u} \in O_{x} M$ : denote its value by $\overline{\bar{f}}^{q}(x)$. We can consider $\overline{\bar{f}}^{q}$ to be an element of $L_{1}(M, \rho)$, and from the invariance of $f^{q}$ under the time shift $\Phi_{T}$ on $O M \times \Omega$, we can deduce that $\overline{\bar{f}}^{q}$ satisfies $P^{T} \overline{\bar{f}}^{q}=\overline{\bar{f}}^{q}$ as elements of $L_{1}(M, \rho)$, where $P^{T} \overline{\bar{f}}^{q}$ is defined by $x \mapsto \int P_{T}(x, d y) \overline{\bar{f}}^{q}(y)$ (so that $P^{t}$ represents heat flow on $M$ ). From this and the ergodicity of $\rho$ we can deduce that $\overline{\bar{f}}^{q}$ is $\rho$-a.s. constant.

COROLlARY 3.4. For any compact Riemannian manifold $M$ there is uniquely defined a set of Lyapunov exponents with associated multiplicities, which are invariants of the Riemannian structure on $M$.

Note. There are also corresponding extrinsic invariants for submanifolds of Euclidean space (see [7]).

4. Bounds on the exponents for general compact Riemannian manifolds. The derivative flow $T \xi_{t}: T O M \rightarrow T O M$ is most easily described using the trivialization

$$
\begin{aligned}
T O M & \rightarrow O M \times \mathbf{R}^{n} \times \mathfrak{o}(n) \\
V & \mapsto(u, \theta(V), \tilde{\omega}(V)), \quad V \in T_{u} O M,
\end{aligned}
$$


where $\mathfrak{o}(n)$ is the Lie algebra of $O(n)$, identified with the space of skew symmetric $n \times n$-matrices, and $\theta, \tilde{\omega}$ are respectively the canonical 1 -form and the connection form on $O M$. The trivialization determines $\mathbf{R}^{n}$ and $o(n)$ valued processes

$$
h_{t}=\theta \circ T \xi_{t}\left(V_{0}\right), \quad A_{t}=\tilde{\omega} \circ T \xi_{t}\left(V_{0}\right),
$$

given $V_{0} \in T O M$. From [10, Corollary VII, 12E] these are determined by the equations

$$
\begin{aligned}
h_{t}= & \theta\left(V_{0}\right)+\int_{0}^{t} A_{s} d B_{s}-\frac{1}{2} \int_{0}^{t} u_{s}^{-1} K\left(v_{s},-\right)^{\#} d s \\
A_{t}= & \tilde{\omega}\left(V_{0}\right)+\int_{0}^{t} u_{s}^{-1} R\left(u_{s} d B_{s}, v_{s}\right) u_{s} \\
& +\frac{1}{2} \int_{0}^{t}\left[u_{s}^{-1} \sum_{i} R\left(u_{s} e_{i}, u_{s} A_{s} e_{i}\right) u_{s}\right] d s \\
& +\frac{1}{2} \int_{0}^{t}\left[u_{s}^{-1} \sum_{i} \nabla R\left(u_{s} e_{i}\right)\left(u_{s} e_{i}, v_{s}\right) u_{s}\right] d s
\end{aligned}
$$

Here $e_{1}, \ldots, e_{n}$ is an orthonormal basis for $\mathbf{R}^{n}, V_{0} \in T_{u_{0}} O M$ and $u_{t}=\xi_{t}\left(u_{0}\right)$ with each frame $u_{t}(\omega)$ considered as an isometry

$$
u_{t}(\omega): \mathbf{R}^{n} \rightarrow T_{x_{t}(\omega)} M, \quad x_{t}=\pi u_{t} .
$$

We use $K: T M \oplus T M \rightarrow \mathbf{R}$ with corresponding $K(v,-)^{\#} \in T M, v \in T M$, and R: $(T M \oplus T M) \oplus T M \rightarrow T M$ to denote the Ricci and full curvature tensor respectively (using Kobayashi and Nomizu's conventions [16]). The scalar curvature will be written as $S: M \rightarrow \mathbf{R}$.

Finally $v_{t}(\omega)=u_{t}(\omega) h_{t}(\omega)=T \pi \circ T \xi_{t}(\omega)\left(V_{0}\right) \in T_{x_{t}(\omega)} M$ is the horizontal component of $T \xi_{t}(\omega)\left(V_{0}\right)$, called the 'derivative process' of Brownian motion on $M$ in $[9, \mathbf{1 0}]$. It is of independent interest, being related to the heat flow of 1-forms [10, Theorem IX2C].

Note that the Stratonovich forms of equations (1a) and (1b) are

$$
\begin{aligned}
& h_{t}=\theta\left(V_{0}\right)+\int_{0}^{t} A_{s} \circ d B_{s} \\
& A_{t}=\tilde{\omega}\left(V_{0}\right)+\int_{0}^{t} u_{s}^{-1} R\left(u_{s} d B_{s}, v_{s}\right) u_{s}
\end{aligned}
$$

which are a direct analogue of the equation for Jacobi fields, and in fact reduce to the first order system corresponding to the Jacobi equation if ' $d B_{s}$ ' is replaced by ' $d s$ ': hence the description 'stochastic Jacobi fields' [20].

We will discuss the behaviour as $t \rightarrow \infty$ of $1 / t \log \left|v_{t}(\omega)\right|$ and use this to obtain estimates on the maximum exponent $\lambda^{\max }$ of $\xi$. First we need to know that $\log \left|v_{t}\right|$ is defined:

LEMMA 4.1. If $\theta\left(V_{0}\right) \neq 0$, then with probability one $v_{t}$ never vanishes for $0 \leq t<\infty$.

Proof. We must show that the subset $Q=O M \times\{0\} \times \mathfrak{o}(n)$ of $O M \times \mathbf{R}^{n} \times \mathfrak{o}(n)$ is unattainable by $\left(u_{0}, h_{0}, A_{0}\right)$ and will use the theory in Friedman [13, vol. II; 12] 
together with the fact that $(u, h ., A$.) are solutions of the systems (1a), (1b), and

$$
d u_{s}=X\left(u_{s}\right) \circ d B_{s} .
$$

Since the problem is purely a local one Friedman's criteria for equations in $\mathbf{R}^{n}$ go over to the manifold case simply by taking local coordinates. Following Friedman take $z=\left(u^{0}, 0, A^{0}\right)$ in $Q$ and use the noise coefficients of (1a), (1b), (1c) to define

$$
\sigma(z): \mathbf{R}^{n} \rightarrow T_{u^{0}} O M \times \mathbf{R}^{n} \times \mathfrak{o}(n)
$$

as

$$
\sigma(z)(e)=\left(X\left(u^{0}\right) e, A^{0} e, 0\right), \quad e \in \mathbf{R}^{n} .
$$

If $E_{i}=\left(0, e_{i}, 0\right)$ for $i=1, \ldots, n$, then $\left\{E_{i}\right\}_{i}$ form a base for the normal space to $Q$ at $z$ and we set $d(z)$ equal to the rank of the diffusion matrix $\alpha$ orthogonal to $S$, i.e. $\alpha=\left(\alpha_{i j}\right)_{i, j}$ where

$$
\alpha_{i j}=\left\langle\sigma(z)^{*} E_{i}, \sigma(z)^{*} E_{j}\right\rangle
$$

However for $e \in \mathbf{R}^{n}$

$$
\left\langle\sigma(z)^{*} E_{i}, e\right\rangle=\left\langle E_{i},\left(X\left(u^{0}\right) e, A^{0} e, 0\right)\right\rangle=\left\langle e_{i}, A^{0} e\right\rangle,
$$

whence $\alpha=-\left(A^{0}\right)^{2}$ and $d(z)=\operatorname{rank}\left(A^{0}\right)^{2}$. Now all points $z$ of $Q$ with $A^{0}=0$ are unattainable by the diffeomorphism property of the flow $\xi_{t}$, and so we can ignore such points and assume $A^{0} \neq 0$. Then since $A^{0}$ is skew symmetric, $d(z) \geq 2$ (take $e$ with $A^{0} e \neq 0$; then $A^{0} A^{0} e \perp A^{0} e$ but $\left\langle A^{0}\left(A^{0}\right)^{2} e, A^{0} e\right\rangle=-\left|\left(A^{0}\right)^{2} e\right| \neq 0$, because $\left\langle\left(A^{0}\right)^{2} e, e\right\rangle=-\left|A^{0} e\right|^{2}$, whence $\left(A^{0}\right)^{2} A^{0} e$ and $\left(A^{0}\right)^{2} e$ are linearly independent).

If $\operatorname{dim} M=2$, then $Q$ has codimension 2 and Theorem 4.2 of [13] gives unattainability immediately. In case $\operatorname{dim} M>2$ we can take a basis in $\mathbf{R}^{n}$ so that $A^{0}$ has matrix consisting of $2 \times 2$ blocks down the diagonal and zeros. The symmmetric matrix of $\alpha \equiv-\left(A^{0}\right)^{2}$ will then have similar structure. In particular $\alpha_{i j}=0$ if $1 \leq i \leq 2$ and $3 \leq j \leq n$ and so 'condition $\mathrm{N}$ ' of Friedman is satisfied and the lemma is proved.

From (1a), the isometric property of each $u_{s}(\omega)$ and $[\mathbf{1 3}]$ we have

$$
\left|h_{t}\right|^{2}=\left|h_{0}\right|^{2}+2 \int_{0}^{t}\left\langle h_{s}, A_{s} d B_{s}\right\rangle+\int_{0}^{t}\left|A_{s}\right|^{2} d s-\int_{0}^{t} K\left(v_{s}, v_{s}\right) d s
$$

where $\left|A_{s}\right|$ refers to the Hilbert-Schmidt norm on $\mathfrak{o}(n)$, i.e. $|A|=\sqrt{\operatorname{trace} A^{*} A}$. Note that for this norm, if $A \in \mathfrak{o}(n)$ and $e \in \mathbf{R}^{n}$, we have

$$
|A e| \leq \frac{1}{\sqrt{2}}|A||e|
$$

with equality if $n=2$.

From (2) and the lemma

$$
\begin{aligned}
\frac{1}{2} \log \left|h_{t}\right|^{2}= & \log \left|h_{0}\right|+\int_{0}^{t}\left\langle h_{s} /\left|h_{s}\right|, A_{s} /\left|h_{s}\right| d B_{s}\right\rangle+\frac{1}{2} \int_{0}^{t}\left|A_{s}\right|^{2} /\left|h_{s}\right|^{2} d s \\
& -\frac{1}{2} \int_{0}^{t} K\left(v_{s} /\left|v_{s}\right|, v_{s} /\left|v_{s}\right|\right) d s-\int_{0}^{t}\left|A_{s} h_{s}\right|^{2} /\left|h_{s}\right|^{4} d s .
\end{aligned}
$$

Therefore by (3)

$$
\frac{1}{2} \log \left|h_{t}\right|^{2} \geq \log \left|h_{0}\right|+M_{t}-\frac{1}{2} \int_{0}^{t} K\left(v_{s} /\left|v_{s}\right|, v_{s} /\left|v_{s}\right|\right) d s
$$


with equality if $n=2$, where

$$
M_{t}=\int_{0}^{t}\left\langle h_{s} /\left|h_{s}\right|, A_{s} /\left|h_{s}\right| d B_{s}\right\rangle .
$$

Now $M_{t}$ is a 1-dimensional stochastic integral and so a time changed Brownian motion on $\mathbf{R}$. Consequently with probability one

giving

$$
\liminf _{t \rightarrow \infty} \frac{1}{t} M_{t} \leq 0 \leq \limsup _{t \rightarrow \infty} \frac{1}{t} M_{t}
$$

$$
\limsup _{t \rightarrow \infty} \frac{1}{t} \log \left|h_{t}\right| \geq-\frac{1}{2} \limsup _{t \rightarrow \infty} \frac{1}{t} \int_{0}^{t} K\left(\frac{v_{s}}{\left|v_{s}\right|}, \frac{v_{s}}{\left|v_{s}\right|}\right) d s .
$$

THEOREM 4.2. Let $\bar{K}: M \rightarrow \mathbf{R}$ be given by $\bar{K}(x)=\sup \left\{K(v, v): v \in T_{x} M,|v|\right.$ $=1\}$. Then

$$
\lambda^{\max } \geq \limsup _{t \rightarrow \infty} \frac{1}{t} \log \left|h_{t}\right| \geq-\frac{1}{2 \operatorname{vol} M} \int_{M} \bar{K}(x) d x .
$$

In particular when $n=2$

$$
\lambda^{\max } \geq-\frac{\pi}{\operatorname{vol} M} \chi(M)
$$

where $\chi$ is the Euler characteristic of $M$.

PROOF. By the ergodic theorem and the fact that the normalized volume element of $M$ is the invariant measure for our Brownian motion $\left\{x_{t}: t \geq 0\right\}$ on $M$ we have

$$
\lim _{t \rightarrow \infty} \frac{1}{t} \int_{0}^{t} \bar{K}\left(x_{s}\right) d s=\int_{M} \bar{K}(x) \frac{d x}{\operatorname{vol} M}
$$

almost surely. This gives (7), and (8) follows by the Gauss-Bonnet theorem and the fact that $K(u, v)=\frac{1}{2} S(x)\langle u, v\rangle_{x}, u, v \in T_{x} M$, when $n=2$.

REMARKS. (i) The theorem is stated in terms of the maximum exponent $\lambda^{\text {max }}$. In fact, since $h_{0}$ is arbitrary, $\lambda^{\max }$ can be replaced by any exponent $\lambda^{r}$ which corresponds to an element $V_{\left(u_{0}, \omega\right)}^{(r)}$ of the filtration which is independent of $\omega$ almost surely (see $[5,15])$.

We can also use the formula for $\left|A_{t}\right|^{2}$ which corresponds to (2). For this define the stochastic process

$$
\tilde{R}:[0, \infty) \times \Omega \rightarrow \mathbf{L}_{s}\left(\Lambda^{2} \mathbf{R}^{n}, \Lambda^{2} \mathbf{R}^{n} ; \mathbf{R}\right),
$$

with values in the space of symmetric bilinear forms on $\Lambda^{2} \mathbf{R}^{n}$, by

$$
\tilde{R}_{s}(\omega)(\alpha, \beta)=\left\langle R\left(u_{s}(\omega) a, u_{s}(\omega) a^{\prime}\right) u_{s}(\omega) b^{\prime}, u_{s}(\omega) b\right\rangle_{x_{s}(\omega)}
$$

when $\alpha=a \wedge a^{\prime}$ and $\beta=b \wedge b^{\prime}$. For $\omega \in \Omega$ let

$$
\tilde{A}_{s}(\omega)=\sum_{i=1}^{n} e_{i} \wedge A_{s}(\omega) e_{i} \in \Lambda^{2} \mathbf{R}^{n}, \quad 0<s<\infty,
$$

and define $\Theta:[0, \infty) \times \Omega \rightarrow \mathbf{L}\left(\mathbf{R}^{n}, \mathbf{L}\left(\Lambda^{2} \mathbf{R}^{n} ; \mathbf{R}\right)\right)$ by

$$
\Theta_{s}(\omega)(a)(\beta)=\nabla K\left(b^{\prime}\right)(b, a)-\nabla K(b)\left(b^{\prime}, a\right)
$$

for $\beta=b \wedge b^{\prime}, a \in \mathbf{R}^{n}$. 
Then by Itô's formula and 1(b) or from Theorem $12 \mathrm{~F}$ and Remark $12 \mathrm{~F}$ of [10] for $n>1$,

$$
\begin{aligned}
\left|A_{t}\right|^{2}= & \left|A_{0}\right|^{2}-2 \int_{0}^{t} \tilde{R}_{s}\left(d B_{s} \wedge h_{s}, \tilde{A}_{s}\right)-\int_{0}^{t} \tilde{R}_{s}\left(\tilde{A}_{s}, \tilde{A}_{s}\right) d s \\
& -\int_{0}^{t} \Theta_{s}\left(h_{s}\right)\left(\tilde{A}_{s}\right) d s+\int_{0}^{t} \sum_{i}\left|R\left(u_{s} e_{i}, u_{s} h_{s}\right)\right|^{2} d s
\end{aligned}
$$

There are two special cases where (2) and (9) simplify: the case $\operatorname{dim} M=2$, and the case of constant curvature.

LEMMA 4.3. (i) When $\operatorname{dim} M=2$,

$$
\begin{aligned}
\left|h_{t}\right|^{2}= & \left|h_{0}\right|^{2}+2 \int_{0}^{t}\left\langle h_{s}, A_{s} d B_{s}\right\rangle+\int_{0}^{t}\left|A_{s}\right|^{2} d s-\frac{1}{2} \int_{0}^{t} S\left(x_{s}\right)\left|h_{s}\right|^{2} d s \\
\left|A_{t}\right|^{2}= & \left|A_{0}\right|^{2}-2 \int_{0}^{t} S\left(x_{s}\right)\left\langle h_{s}, A_{s} d B_{s}\right\rangle-\int_{0}^{t} S\left(x_{s}\right)\left|A_{s}\right|^{2} d s \\
& -\int_{0}^{t} d S\left(u_{s} A_{s} h_{s}\right) d s+\int_{0}^{t} \frac{1}{2} S\left(x_{s}\right)^{2}\left|h_{s}\right|^{2} d s
\end{aligned}
$$

(ii) When $M$ has constant sectional curvature $k$,

$$
\begin{aligned}
\left|h_{t}\right|^{2}= & \left|h_{0}\right|^{2}+2 \int_{0}^{t}\left\langle h_{s}, A_{s} d B_{s}\right\rangle+\int_{0}^{t}\left|A_{s}\right|^{2} d s-k(n-1) \int_{0}^{t}\left|h_{s}\right|^{2} d s \\
\left|A_{t}\right|^{2}= & \left|A_{0}\right|^{2}-4 k \int_{0}^{t}\left\langle h_{s}, A_{s} d B_{s}\right\rangle-2 k \int_{0}^{t}\left|A_{s}\right|^{2} d s \\
& +2 k^{2}(n-1) \int_{0}^{t}\left|h_{s}\right|^{2} d s
\end{aligned}
$$

PROOF. (i) Fix $s>0$. We can take an orthonormal basis $e_{1}, e_{2}$ for $\mathbf{R}^{2}$ with $A_{s} e_{1}=-\alpha_{s} e_{2}, A e_{2}=\alpha_{s} e_{1}$ for some $\alpha_{s} \in \mathbf{R}$. We have, for any frame $u$ at $x \in M$,

$$
S(x)=2\left\langle R\left(u e_{1}, u e_{2}\right) u e_{2}, u e_{1}\right\rangle \text { and } \tilde{A}_{s}=-2 \alpha_{s} e_{1} \wedge e_{2}
$$

Thus for $e=\beta_{1} e_{1}+\beta_{2} e_{2} \in \mathbf{R}^{2}$ and $h \in \mathbf{R}^{2}$

$$
\tilde{R}_{s}\left(e \wedge h, \tilde{A}_{s}\right)=\left(e_{1} h_{2}-e_{2} h_{1}\right)\left(-2 \alpha_{s}\right) \tilde{R}_{s}\left(e_{1} \wedge e_{2}, e_{1} \wedge e_{2}\right)=\left\langle A_{s} e, h\right\rangle S(x)
$$

and

$$
\tilde{R}_{s}\left(\tilde{A}_{s}, \tilde{A}_{s}\right)=2 \alpha^{2} S(x)=\left|A_{s}\right|^{2} S(x)
$$

while $K(u, v)=\frac{1}{2} S(x)\langle u, v\rangle, u, v \in T_{x} M$, so that

$$
\begin{aligned}
\Theta_{s}(h)\left(\tilde{A}_{s}\right) & =-2 \alpha \Theta_{s}(h)\left(e_{1} \wedge e_{2}\right) \\
& =-\alpha_{s} h_{1} d S\left(u_{s} e_{2}\right)+\alpha_{s} h_{2} d S\left(u_{s} e_{1}\right)=\alpha S\left(u_{s} A_{s} h\right)
\end{aligned}
$$

Finally

$$
\begin{aligned}
\sum_{i}\left|R\left(u_{s} e_{i}, h_{s}\right)\right|^{2} & =\sum_{i} 2\left\langle R\left(u_{s} e_{i}, h_{s}\right) u_{s} e_{2}, u_{s} e_{1}\right\rangle^{2} \\
& =2\left|h_{s}\right|^{2} \frac{1}{4} S(x)^{2} .
\end{aligned}
$$


(ii) Equations (11a) and (11b) follow from (2) and (9) and the identities $R(X, Y) Z$ $=k\left(\langle Z, Y\rangle_{x} X-\langle Z, X\rangle_{x} Y\right)$ and $K(X, X)=(n-1) k|X|_{x}^{2}$ for $X, Y, Z \in T_{x} M$, when $M$ has constant curvature $k$ (see $[16$, p. 203]).

The frame bundle of the universal cover of a manifold of constant curvature has a natural Lie group structure, see $\S 5$ below. The following proposition reflects the bi-invariance of its Killing form, its proof is immediate from (11a, b).

PROPOSITION 4.4. For a manifold of constant sectional curvature $k$ we have, almost surely,

$$
\left|A_{t}\right|^{2}+2 k\left|h_{t}\right|^{2}=\left|A_{0}\right|^{2}+2 k\left|h_{0}\right|^{2}, \quad 0 \leq t<\infty .
$$

When $k>0$ we can take $|A|^{2}+2 k|h|^{2}$ to be the norm of $(A, h)$ to see (using Proposition 2.5)

COROLLARY 4.4.1. All the exponents are zero for a manifold of constant positive sectional curvature.

In dimension 2 there is a generalization of equation (12) to the case of nonconstant curvature.

Proposition 4.5. If $\operatorname{dim} M=2$, there is the Stratonovich equation

$$
d\left|A_{t}\right|^{2}+S\left(x_{s}\right) \circ d\left|h_{t}\right|^{2}=0
$$

and the Itô equation

$$
\begin{aligned}
\left|A_{t}\right|^{2}+S\left(x_{s}\right)\left|h_{t}\right|^{2}= & \left|A_{0}\right|^{2}+S\left(x_{0}\right)\left|h_{0}\right|^{2}+\int_{0}^{t}\left|h_{s}\right|^{2} d s\left(u_{s} d B_{s}\right) \\
& +\frac{1}{2} \int_{0}^{t}\left|h_{s}\right|^{2} \Delta S\left(x_{s}\right) d s+\int_{0}^{t} d S\left(u_{s} A_{s} h_{s}\right) d s
\end{aligned}
$$

Proof. From (10a), (10b) and the Itô formula for $S\left(x_{s}\right)$

$$
\begin{aligned}
\left|A_{t}\right|^{2}+S\left(x_{t}\right)\left|h_{t}\right|^{2}= & \left|A_{0}\right|^{2}+S\left(x_{0}\right)\left|h_{0}\right|^{2}-\int_{0}^{t} d S\left(u_{s} A_{s} h_{s}\right) d s \\
& +\int_{0}^{t}\left|h_{s}\right|^{2} d S\left(u_{s} d B_{s}\right)+\frac{1}{2} \int_{0}^{t}\left|h_{s}\right|^{2} \Delta S\left(x_{s}\right) d s \\
& +2 \int_{0}^{t} \sum_{i}\left\langle h_{s}, A_{s} e_{i}\right\rangle d S\left(u_{s} e_{i}\right) d s
\end{aligned}
$$

This gives (14). The right-hand side is just the Stratonovich expression

$$
\left|A_{0}\right|^{2}+S\left(x_{0}\right)\left|h_{0}\right|^{2}+\int_{0}^{t}\left|h_{s}\right|^{2} \circ d\left(S\left(x_{s}\right)\right)
$$

so that (13) follows by integration by parts. Alternatively (13) can be derived directly from $\left(1 a^{\prime}\right)$ and $\left(1 b^{\prime}\right)$.

Proposition 4.6 (Positive CuRVATURe UPPERBound). If $\operatorname{dim} M=2$ and $M$ has positive curvature at all points, then

$$
\lambda^{\max } \leq \frac{1}{4} \int_{M}\left\{\frac{|\nabla S(x)|}{\sqrt{2} \sqrt{S(x)}}-\frac{|\nabla S(x)|^{2}}{S(x)^{2}}\right\} \frac{d x}{\operatorname{vol} M}
$$


ProOF. Since $S(x)>0$ for all $x$ we can take $\sqrt{\left\{\left|A_{t}\right|^{2}+S\left(x_{t}\right)\left|h_{t}\right|^{2}\right\}}$ to be the norm of $\left(h_{t}, A_{t}\right)$. By (14)

$$
\begin{aligned}
& \frac{1}{2} \log \left(\left|A_{t}\right|^{2}+S\left(x_{t}\right)\left|h_{t}\right|^{2}\right)=\frac{1}{2} \log \left(\left|A_{0}\right|^{2}+S\left(x_{0}\right)\left|h_{0}\right|^{2}\right) \\
& \quad+\frac{1}{2} \int_{0}^{t} \frac{\left|h_{s}\right|^{2} d S\left(u_{s} d B_{s}\right)}{\left|A_{s}\right|^{2}+S\left(x_{s}\right)\left|h_{s}\right|^{2}}+\frac{1}{2} \int_{0}^{t} \frac{d S\left(u_{s} A_{s} h_{s}\right)}{\left|A_{s}\right|^{2}+S\left(x_{s}\right)\left|h_{s}\right|^{2}} d s \\
& \quad+\frac{1}{4} \int_{0}^{t} \frac{\left|h_{s}\right|^{2}}{\left|A_{s}\right|^{2}+S\left(x_{s}\right)\left|h_{s}\right|^{2}} \Delta S\left(x_{s}\right) d s-\frac{1}{2} \int_{0}^{t} \frac{\left|h_{s}\right|^{4}\left|\nabla S\left(x_{s}\right)\right|^{2}}{\left(\left|A_{s}\right|^{2}+S\left(x_{s}\right)\left|h_{s}\right|^{2}\right)^{2}} d s
\end{aligned}
$$

Now

$$
\begin{aligned}
\left|d S\left(u_{s} A_{s} h_{s}\right)\right| & \leq \frac{\left|\nabla S\left(x_{s}\right)\right|}{\sqrt{S\left(x_{s}\right)}} \frac{1}{\sqrt{2}}\left|A_{s}\right|\left(\sqrt{S\left(x_{s}\right)}\left|h_{s}\right|\right) \\
& \leq\left|\nabla S\left(x_{s}\right)\right| S\left(x_{s}\right)^{-1 / 2} \frac{1}{2 \sqrt{2}}\left(\left|A_{s}\right|^{2}+S\left(x_{s}\right)\left|h_{s}\right|^{2}\right) .
\end{aligned}
$$

Therefore the ergodic theorem together with the boundedness of the coefficients of the Itô integral imply that

$$
\lambda^{\max } \leq \frac{1}{4 \sqrt{2}} \int_{M} \frac{|\nabla S(x)|}{\sqrt{S(x)}} \frac{d x}{\operatorname{vol} M}+\frac{1}{4} \int_{M} \frac{\Delta S(x)}{S(x)} \frac{d x}{\operatorname{vol} M}
$$

and the result follows by the divergence theorem and the identity

$$
\Delta \log S(x)=\frac{\Delta S(x)}{S(x)}-\frac{|\nabla S(x)|^{2}}{S(x)^{2}} .
$$

Since $\lambda^{\max }$ is always nonnegative by Proposition 2.5, the proposition yields the differential-geometric inequality for all compact surfaces $M$ of positive curvature:

$$
\int_{M} \frac{|\nabla S(x)|^{2}}{S(x)^{2}} d x \leq \frac{1}{\sqrt{2}} \int_{M} \frac{|\nabla S(x)|}{\sqrt{S(x)}} d x .
$$

The right-hand side is dominated by

$$
\frac{1}{\sqrt{2}} \sqrt{\int_{M} \frac{|\nabla S(x)|^{2}}{S(x)^{2}}} d x \sqrt{\int_{M} S(x) d x}
$$

whence

$$
\int_{M} \frac{|\nabla S(x)|^{2}}{S(x)^{2}} d x \leq \frac{1}{2} \int_{M} S(x) d x=2 \pi \chi(M)=4 \pi
$$

if $M$ is orientable, by the Gauss-Bonnet theorem and the fact that such $M$ are topologically $S^{2}$. From (15) and (18) we obtain the rather crude bound

COROLLARY 4.6.1. If $M$ is an orientable surface of strictly positive curvature, then

$$
0 \leq \lambda^{\max } \leq \pi / \operatorname{vol} M
$$

However it may well be that $\lambda^{\max }=0$ for all such $M$. 
5. The case of constant curvature. A simply connected manifold $N$ of constant curvature is isometric to a homogeneous space $G / S O(n)$, where $G=$ $S O(n+1)$ for positive curvature and is the connected component of the identity in the Lorentz group $O(1, n)$ for $n$ space dimensions and one time dimension in the case of negative curvature. Moreover the bundle $G \rightarrow G / S O(n)$ can be identified with the special orthogonal frame bundle of $N[\mathbf{1 7}, \mathrm{pp} .264,268]$. As observed by Malliavin and Malliavin $[\mathbf{1 8}, 19]$ for more general symmetric spaces under this identification the canonical flow is given by the S.D.E. on $G$

$$
d u=\tilde{X}(u) \circ d B
$$

where $\tilde{X}$ is left invariant. The flow becomes

$$
\xi_{t}(\omega): G \rightarrow G, \quad \xi_{t}(\omega)(u)=u \cdot g_{t}(\omega),
$$

where $\left\{g_{t}: t \geq 0\right\}$ is the solution of (21) with $g_{0}$ the identity in $G$.

Since this gives such a precise version of the canonical flow it can be used to give a precise description of its long time behaviour and Lyapunov spectrum [2, 18, 19]. For example since $S O(n+1)$ has a bi-invariant Riemannian metric the canonical flow for manifolds of positive curvature consists of isometries (for this metric transferred to $O M$ ) and so as seen in Corollary 3.4.1 all the exponents are zero. More generally the bi-invariance of the Cartan-Killing form shows that (12) is sure for this version of the flow, rather than just almost sure.

Rather than follow this approach we will restrict ourselves to the case of surfaces of constant negative curvature and show by direct arguments that the stable manifold structure is essentially the same as for the geodesic flow. However we should point out that we were first led to believe this by Guivarc' $h$ who argued using the homogeneous space structure.

In the following theorem we define the stable manifolds for the canonical flow:

THEOREM 5.1. For each $(\mathfrak{u}, \omega) \in \Gamma$ and each strïctly negative Lyapunov exponent $\lambda^{i}$, the set $\mathcal{V}_{(u, \omega)}^{(i)}$ given by

$$
\left\{\mathfrak{v}: \limsup _{t \rightarrow \infty} \frac{1}{t} \log d\left(\xi_{t}(\omega) \mathfrak{u}, \xi_{t}(\omega) \mathfrak{v}\right) \leq \lambda^{i}\right\}
$$

is the image of the corresponding element $V_{(\mathfrak{u}, \omega)}^{(i)}$ of the filtration under a smooth immersion which is tangent to the identity at $u$. (The set $\mathcal{V}_{(\mathfrak{u}, \omega)}^{(i)}$ is called the stable manifold at $(u, \omega)$ corresponding to $\lambda^{i}$.)

ProOF. The proof is clear from [3, Theorem 2.2.2].

Without loss of generality suppose that $M$ has constant curvature -1 . It can therefore be isometrically covered by the hyperbolic plane $N$ and it is easily seen that any Lyapunov structure for the canonical flow of $N$ will project to one on $M$ via the covering map. We shall therefore work with $N$ and express our answers in terms of isometries of $N$ and in particular the horospheres (see [1, Appendix 21]).

It will be convenient to consider both standard models of $N$. The unit disc model represents $N$ as $D=\left\{(x, y): x^{2}+y^{2}<1\right\}$ with Riemannian metric $\left[1-\left(x^{2}+y^{2}\right)\right]\left(d x^{2}+d y^{2}\right)$. Here the unit circle can be considered as the 'circle at infinity' $N_{\infty}$. The upper half plane model represents $N$ as $U^{c}=\{(x, y): y>0\}$ 
with metric $y^{-2}\left(d x^{2}+d y^{2}\right)$ and we use the superscript $c$ to indicate that we have identified a particular point $c$ of $N_{\infty}$ with the point at infinity in the upper half plane (thus $N_{\infty}-\{c\}$ corresponds to the $x$-axis). Note that an isometry of $N$ with $U^{c}$ is completely determined by the choice of $c \in N_{\infty}$ and the inverse image of $(0,1) \in U^{c}$. An isometry of $N$ with $D$ is determined by the inverse image $d$ of $(0,0) \in D$ and the angle of inclination of a fixed half-ray from $d$.

In the model $U^{c}$ we will consider isometries of the following type.

TYPE I. Horizontal translations of $U^{c}$.

TYPE II. The linear expansions, $z \mapsto \alpha z$, of $U^{c}$ for $\alpha>0$.

In the model $D$ we will consider:

TYPE III. Rotations of $D$ about the origin.

Using these isometries it is rather easy to see that $N$ and its frame bundle have the homogeneous space structure mentioned before:

LEMMA 5.2. For any $u, v \in O N$ there is a unique isometry $g$ which sends $u$ to v, i.e. $O_{g}(u)=v$.

PROOF. After a suitable isometry with $U^{c}$ we can obtain isometries of Type I which transform $u$ and $v$ to frames at $(0,1)$. Then an isometry with $D$ sending $(0,1)$ to $(0,0)$ enables these frames to be aligned by a rotation. Uniqueness follows from the fact that the only isometries fixing $(0,0)$ in the $D$ model are the rotations.

It will be convenient to use the Buseman functions of $N$ : if $p \in N$ we can identify each $c \in N_{\infty}$ with the $\frac{1}{2}$-ray $\gamma$, parametrized by arc length which sets off from $p$ in the direction $c$, and we define $\beta_{p}(c,-): N \rightarrow \mathbf{R}$ by

$$
\beta_{p}(c, z)=\lim _{t \rightarrow \infty}[t-d(z, \gamma(t))] .
$$

We have the following standard and easily verified result.

LEMMA 5.3. The function $\beta_{p}$ exists and is finite on $N$. In the model $U^{c}$ it is given by $\beta_{p}(c, z)=\log y$ when $p=(0,1)$ and $z=(x, y)$. Thus it is the hyperbolic distance of $z$ from the line $y=1$. 1 ,

LEMMA 5.4. Let $\left\{z_{t}: t \geq 0\right\}$ be a Brownian motion on $N$. Then with probability

(i) $z_{\infty}(\omega)=\lim _{t \rightarrow \infty} z_{t}(\omega) \in N_{\infty}$ exists and

(ii) $\lim _{t \rightarrow \infty}(1 / t) \beta_{z_{0}}\left(z_{\infty}(\omega), z_{t}(\omega)\right)=\frac{1}{2}$.

ProOF. Part (i) is a special case of Prat's result for nonconstant negative curvature, see $[\mathbf{2 2}, \mathbf{2 3}, \mathbf{2 6}]$, and is easily seen in our case by working in either of the models $D$ or $U^{c}$.

For (ii) it suffices to show that if $c \in N_{\infty}$, then

$$
\mathbf{P}\left\{\frac{1}{t} \beta_{z_{0}}\left(c, z_{t}(\omega)\right) \rightarrow \frac{1}{2} \mid z_{\infty}(\omega)=c\right\}=1
$$

and for this we use the Doob $h$-transform to condition $z$ to tend to $c$. In fact we only need to use the simplest example of $h$-transform theory because in the $D$ model the Brownian motion of $N$ is just a time changed Euclidean Brownian motion stopped at $S^{1}$ and time changing commutes with our conditioning. As described in [8,2X9] 
the Euclidean B.M. in $D$ conditioned to exit at $a \in S^{1}$ is the $h$-Brownian motion where $h$ is given by the Poisson kernel

$$
h(z)=\left(1-|z|^{2}\right) /|a-z|^{2}, \quad|z|<1,
$$

i.e. it has the law of the diffusion process with generator $\frac{1}{2} \Delta+\nabla \log h$. Therefore Brownian motion on $N$ conditioned to tend to $a$ as $t \rightarrow \infty$ is the diffusion with generator $\frac{1}{2} \Delta_{N}+\nabla_{N} \log h$, where $\Delta_{N}$ and $\nabla_{N}$ refer to the hyperbolic metric. In the $U^{c}$ model (when $c$ is mapped to $\infty$ ) this diffusion can be given by solutions of the S.D.E.

$$
d x_{t}=y_{t} d B_{t}^{1}, \quad d y_{t}=y_{t} d B_{t}^{2}+y_{t} d t
$$

for $\left\{\left(B_{t}^{1}, B_{t}^{2}\right): t \geq 0\right\}$ a B.M. on $\mathbf{R}^{2}$. (Note that the Christoffel symbols in the $U^{c}$ model are given in the proof of Theorem 5.7.) Then $y_{t}=y_{0} \exp \left(B_{t}^{2}+\frac{1}{2} t\right)$ and the result follows by Lemma 5.3 .

THEOREM 5.5. Take $u \in O N$. Then for almost all $\omega \in \Omega$ we have the following:

We can take $c=\lim _{t \rightarrow \infty} \pi \xi_{t}(\omega) u \in N_{\infty}$ and let $\mathcal{V}_{(u, \omega)}$ be the 1-dimensional submanifold of $O N$ given by $\left\{O_{g}(u): g\right.$ a Type I isometry (horizontal translation) in $\left.U^{c}\right\}$. Then for any $u^{\prime} \in \mathcal{V}_{(u, \omega)}$ we have

$$
\lim _{t \rightarrow \infty} \frac{1}{t} \log d\left(\xi_{t}(\omega) u, \xi_{t}(\omega) u^{\prime}\right)=-\frac{1}{2}
$$

and for all other frames $u^{\prime}$

$$
\varliminf_{t \rightarrow \infty} \frac{1}{t} \log d\left(\xi_{t}(\omega) u, \xi_{t}(\omega) u^{\prime}\right) \geq 0 .
$$

ProOF. Choose $\omega \in \Omega$ so that the conclusions of Lemmas 2.2 and 4.3 are valid for $z_{t}(\omega)=\pi \xi_{t}(\omega) u$. Then $c=z_{\infty}(\omega)$ exists and we will work in $U^{c}$.

We need only consider oriented frames and a frame at $(x, y)$ can be identified with a tangent vector to $U^{c}$ at $(x, y)$ of unit Euclidean length: with this identification we will write frames as $(x, y, \lambda) \in U^{c} \times S^{1}$. This also determines a new metric $\tilde{d}$ on the space of oriented frames, namely the product of the Euclidean metric on $U^{c}$ with the standard one on $S^{\mathbf{1}}$. This will be equivalent to the standard metric on the frame bundle over each compact subset of $U^{c}$, in particular over the set

$$
W=\left\{(x, y) \in U^{c}:|x|+|1-y| \leq \frac{1}{2}\right\} .
$$

Write $\xi_{t}(\omega) u=\left(x_{t}, y_{t}, \lambda_{t}\right)$. Then if $v \in \mathcal{V}_{(u, \omega)}$ there exists $a \in \mathbf{R}$ with

$$
\xi_{t}(\omega) v=\left(x_{t}+a, y_{t}, \lambda_{t}\right), \quad t \geq 0 .
$$

Using isometries of Types I and II

$$
d\left(\xi_{t}(\omega) u, \xi_{t}(\omega) v\right)=d\left(\left(0, y_{t}, \lambda_{t}\right),\left(a, y_{t}, \lambda_{t}\right)\right)=d\left(\left(0,1, \lambda_{t}\right),\left(\frac{a}{y_{t}}, 1, \lambda_{t}\right)\right)
$$

But for sufficiently large $t$ both $(0,1)$ and $\left(a y_{t}^{-1}, 1\right)$ lie in $W$ over which $d$ and $\tilde{d}$ are equivalent. Now

$$
\tilde{d}\left(\left(0,1, \alpha_{t}\right),\left(\frac{a}{y_{t}}, 1, \alpha_{t}\right)\right)=\frac{|a|}{\left|y_{t}\right|}
$$


and

$$
\lim _{t \rightarrow \infty} \frac{1}{t} \log \frac{|a|}{\left|y_{t}\right|}=-\frac{1}{2}
$$

by Lemmas 5.3 and 5.4(ii). This proves (22).

If $u^{\prime} \notin \mathcal{V}_{(u, \omega)}$, but in the representation $U^{c}$ is obtained from $u$ by an isometry of Type II, i.e., for some $\alpha>0, \alpha \neq 1, u^{\prime}=\left(\alpha x_{0}, \alpha y_{0}, \lambda_{0}\right)$ for $u=\left(x_{0}, y_{0}, \lambda_{0}\right)$ then $\xi_{t}(\omega) u^{\prime}=\left(\alpha x_{t}, \alpha y_{t}, \lambda_{t}\right)$ and

$$
d\left(\xi_{t}(\omega) u^{\prime}, \xi_{t}(\omega) u\right) \geq d\left(\left(0, \alpha y_{t}\right),\left(0, y_{t}\right)\right)=d((0, \alpha),(0,1))
$$

so that (23) holds.

On the other hand if $u^{\prime} \notin \mathcal{V}_{(u, \omega)}$ but is not in this class, then $\lim _{t \rightarrow \infty} \xi_{t}(\omega) u^{\prime} \neq c$, as can be seen from the proof of Lemma 5.2. Consequently

$$
d\left(\xi_{t}(\omega) u, \xi_{t}(\omega) u^{\prime}\right) \geq d\left(\pi \xi_{t}(\omega) u, \pi \xi_{t}(\omega) u^{\prime}\right) \geq \beta_{p}\left(c, \pi \xi_{t}(\omega) u\right)
$$

for all sufficiently large $t$ and any $p \in N$, by Lemma 5.3 . Thus by 5.4

$$
\varliminf_{t \rightarrow \infty} \frac{1}{t} d\left(\xi_{t}(\omega) u, \xi_{t}(\omega) u^{\prime}\right) \geq \frac{1}{2}
$$

giving (23) again.

REMARK. In fact we can easily prove Baxendale's result that if $\lim _{t \rightarrow \infty} \pi \xi_{t}(\omega) u$ $\neq \lim _{t \rightarrow \infty} \pi \xi_{t}(\omega) u^{\prime}$, then a.s.

$$
\lim _{t \rightarrow \infty} \frac{1}{t} d\left(\xi_{t}(\omega) u, \xi_{t}(\omega) u^{\prime}\right)=1
$$

although we leave it to the reader to show that if $u^{\prime}$ comes from $u$ by a nontrivial isometry of Type II, then a.s.

$$
\lim _{t \rightarrow \infty} \frac{1}{t} d\left(\xi_{t}(\omega) u, \xi_{t}(\omega) u^{\prime}\right)=0 .
$$

See [2] for more precise results.

PROOF OF (24). It suffices to show that for $d_{t}=d\left(\pi \xi_{t}(\omega) u, \pi \xi_{t}(\omega) u^{\prime}\right)$ we have $\lim _{t \rightarrow \infty} d_{t} / t=1$ since the fibres of the frame bundle all have the same diameter. However if $p \in N$ is some point and $r_{t}=d\left(\pi \xi_{t}(\cdot) u, p\right)$ and $r_{t}^{\prime}=d\left(\pi \xi_{t}(\cdot) u^{\prime}, p\right)$, then a.s.

$$
\lim _{t \rightarrow \infty} r_{t} / t=\lim _{t \rightarrow \infty} r_{t}^{\prime} / t=\frac{1}{2}
$$

as in [22].

Thus $\varlimsup_{t \rightarrow \infty} \frac{1}{t} d_{t} \leq \varlimsup_{t \rightarrow \infty} \frac{1}{t}\left(r_{t}+r_{t}^{\prime}\right)=1$. On the other hand, since $\beta_{p}(c, z)$ is the hyperbolic distance of $z$ from the horosphere through $p$ and $c$ we see that with probability 1 we can take points $q$ and $q^{\prime}$ so that

$$
d_{t}(\omega) \geq \beta_{q}\left(\pi \xi_{\infty}(\omega) u, \pi \xi_{t}(\omega) u\right)+\beta_{q^{\prime}}\left(\pi \xi_{\infty}(\omega) u^{\prime}, \pi \xi_{t}(\omega) u^{\prime}\right),
$$

and consequently by Lemma 5.4

$$
\lim _{t \rightarrow \infty} \frac{1}{t} d_{t}(\omega) \geq 1
$$

as required.

We now have a straightforward technical lemma. 
LEMMA 5.6. For given $\left(x_{0}, y_{0}\right) \in U^{c}$ let $\left\{\left(x_{t}, y_{t}\right): t \geq 0\right\}$ be the solution of the system

$$
d x_{t}=y_{t} d B_{t}^{1}, \quad d y_{t}=y_{t} d B_{t}^{2}+y_{t} d t
$$

driven by a 2-dimensional Brownian motion $\left\{\left(B_{t}^{1}, B_{t}^{2}\right): t \geq 0\right\}$. Then

$$
\varlimsup_{t \rightarrow \infty} \frac{1}{t} \log \left|\frac{x_{t}}{y_{t}}\right|=0 \quad \text { a.s. }
$$

PROOF. Set $\theta_{t}=x_{t} / y_{t}^{-1}$. Then

$$
\theta_{t}=\exp \left\{-B_{t}^{1}-\frac{1}{2} t\right\} \int_{0}^{t} e^{B_{s}^{2}+s / 2} d B_{s}^{1}
$$

so that

$$
\lim _{t \rightarrow \infty} \frac{1}{t} \log \left|\theta_{t}\right|=-\frac{1}{2}+\lim _{t \rightarrow \infty} \frac{1}{t} \log \left|\int_{0}^{t} e^{B_{s}^{2}+s / 2} d B_{s}^{1}\right|
$$

provided it exists.

Write $B$ for $B^{2}$ and set

$$
w_{t}=\int_{0}^{t} e^{B_{s}+s / 2} d B_{s}^{1}
$$

There is a 1-dimensional Brownian motion, $\tilde{B}$ say, such that $\tilde{B}_{r(t)}=w_{t}$, where

$$
r(t)=\int_{0}^{t} e^{2 B_{s}+s} d s
$$

Take $\varepsilon>0$, by Khintchine's law of the iterated logarithm, it follows that with probability one there exists $T_{0}$ with

$$
\left|w_{t}\right| \leq(1+\varepsilon) \sqrt{\{2 r(t) \log \log r(t)\}}
$$

for $t \geq T_{0}$. For such $t$

$$
\frac{1}{t} \log w_{t} \leq \frac{1}{t} \log (1+\varepsilon)+\frac{1}{2 t} \log 2 r(t)+\frac{1}{2 t} \log \log \log r(t) .
$$

We claim $\varlimsup_{t \rightarrow \infty}(1 / 2 t) \log 2 r(t) \leq \frac{1}{2}$. From this we see by (28) and (31) that

$$
\varlimsup \frac{1}{t} \log \left|\theta_{t}\right| \leq-\frac{1}{2}+\varlimsup \frac{1}{t} \log \left|w_{t}\right| \leq 0
$$

while $\varlimsup \lim \left|\theta_{t}\right|=\infty$ since $d \theta_{t}=d B_{t}^{1}-z_{t} d B_{t}^{2}$ so that equality holds in (27) as required. To prove the claim use Chung's log log law:

$$
\mathbf{P}\left\{\varlimsup_{t \rightarrow \infty} \sup _{0 \leq s \leq t} \frac{\left|B_{s}\right|}{\sqrt{(2 t \log \log t)}}=1\right\}=1,
$$

to see that with probability one there exists $T_{1}$ such that for $t \geq T_{1}$

$$
\begin{aligned}
r(t) & \leq \int_{0}^{t} \exp \{2(1+\varepsilon) \sqrt{(2 t \log \log t)}+s\} d s \\
& =\left(e^{t}-1\right) \exp \{2(1+\varepsilon) \sqrt{(2 t \log \log t)}\}
\end{aligned}
$$


so that

$$
\frac{1}{2 t} \log 2 r(t) \leq \frac{1}{2}+\frac{1}{t}(1+\varepsilon) \sqrt{(2 t \log \log t)} \rightarrow \frac{1}{2} \quad \text { as } t \rightarrow \infty .
$$

Our final result describes the Lyapunov spectrum for the canonical flow of $N$, and so for any complete surface of constant curvature -1 by taking the universal cover:

THEOREM 5.7. The Lyapunov exponents are $-\frac{1}{2}, 0, \frac{1}{2}$, with corresponding filtration $V_{(u, \omega)}^{(1)} \subset V_{(u, \omega)}^{(2)} \subset V_{(u, \omega)}^{(3)}=T_{u} O(M)$ at a given frame $u$ which can be described with probability one in terms of $c=\lim _{t \rightarrow \infty} \pi \xi_{t}(\omega) u$ as follows:

(a) $V_{(u, \omega)}^{(1)}$ is the tangent space to the orbit of Type I isometries (horizontal translations) in the model $U^{c}$;

(b) $V_{(u, \omega)}^{(2)}$ is the span of $V_{(u, \omega)}^{(1)}$ and the tangent space to the orbit of Type II isometries (multiplication by some $\alpha>0$ ) in the model $U^{c}$.

The stable manifold through $u$ corresponding to the negative exponent is $\mathcal{V}_{(u, \omega)}$ as described in Theorem 5.6.

PROOF. The most direct method to see that the top exponent is $\frac{1}{2}$ is to go back to equation (5) of the last section. In our case it gives

$$
\log \left|h_{t}\right|=\log \left|h_{0}\right|+M_{t}-\frac{1}{2} t
$$

where $M_{t}=\int_{0}^{t}\left\langle h_{s} /\left|h_{s}\right|, A_{s} /\left|h_{s}\right| d B_{s}\right\rangle$. This time we can apply equation (12) to see that $M_{t} / t \rightarrow 0$ as $t \rightarrow \infty$, so that $t^{-1} \log \left|h_{t}\right| \rightarrow-\frac{1}{2}$ as $t \rightarrow \infty$. The result follows by using (12) again.

Since the weighted sum of the exponents is 0 there must be at least one negative exponent. Since such have corresponding stable manifolds the only possibility is $-\frac{1}{2}$ by Theorem 4.4 with $V_{(u, \omega)}^{(1)}$ tangent to the stable manifold $V_{(u, \omega)}$.

Now $\operatorname{dim} V_{(u, \omega)}^{(1)}=1$ and $\operatorname{dim} V_{(u, \omega)}^{(3)}=3$ so that to get a weighted sum of 0 there must be an exponent 0 , and it only remains to identify the two-dimensional subspace $V_{(u, \omega)}^{(2)}$ as that described in (b). To do this work in the model $U^{c}$ but rather than using the notation of the proof of Theorem 4.4 we will use the standard procedure of embedding the orthonormal frame bundle of $N$ in its full frame bundle and identifying this with the product $U^{c} \times \mathrm{GL}(2, \mathbf{R})$. Thus a typical frame is denoted by $(x, y, L)$, where $L \subset \mathrm{GL}(2, \mathbf{R})$ and we will take $\left(x_{0}, y_{0}, L_{0}\right)$ as our initial frame $u$. Also the tangent space at $u$ to Type II orbit through $u$ is spanned by $v$ for $v$ represented by $v=\left(x_{0}, y_{0}, L_{0}\right)$. Then

$$
\begin{aligned}
T_{u} \xi_{t}(\omega) v & =\left.\frac{d}{d s} \xi_{t}(\omega)\left((1+s) x_{0},(1+s) y_{0},(1+s) L_{0}\right)\right|_{s=0} \\
& =\left.\frac{d}{d s}\left((1+s) x_{t},(1+s) y_{t},(1+s) L_{t}\right)\right|_{s=0} \\
& =\left(x_{t}, y_{t}, L_{t}\right) .
\end{aligned}
$$

The horizontal component $h_{t}$, say, of $T_{u} \xi_{t}(\omega) v$ is therefore given by $h_{t}=\left(x_{t}, y_{t}\right)$ so that, using the Riemannian norm,

$$
\left|h_{t}\right|=\sqrt{\left(1+\left(x_{t} / y_{t}\right)^{2}\right)}
$$


By Lemma 5.6,

$$
0 \leq \varliminf \frac{1}{t}\left|h_{t}\right| \leq \varlimsup \frac{1}{t}\left|h_{t}\right|=0
$$

Thus

$$
\lim _{t \rightarrow \infty} \frac{1}{t} \log \left|h_{t}\right|=0
$$

We want to show

$$
\lim _{t \rightarrow \infty} \frac{1}{t} \log \left|T_{u} \xi_{t}(\omega) v\right|=0 .
$$

In fact this follows from (33) using the sure invariance of the Killing forms, described at the beginning of this section, which allows us to apply (12) to our situation and so see that the vertical component $A_{t}$, say, of $T_{u} \xi_{t}(\omega) v$ grows at the same rate as $h_{t}$. Alternatively we can compute $A_{t}$ explicitly as follows (by $A_{t}$ we mean the element of $o(2)$ obtained using the connection form as in the last section):

The Christoffel symbols of $N$ in the chart $U^{c}$ at the point $(x, y)$ are

$$
\Gamma_{11}^{1}=\Gamma_{12}^{2}=\Gamma_{21}^{2}=\Gamma_{22}^{1}=0, \quad \Gamma_{11}^{2}=\frac{1}{y}, \quad \Gamma_{12}^{1}=\Gamma_{21}^{1}=\Gamma_{22}^{2}=-\frac{1}{y} .
$$

Therefore, since the metric is conformally Euclidean and $L_{t}$ is an orthonormal frame

$$
\left(A_{t}\right)_{i}^{k}=\left(\sum_{j=1}^{2} \Gamma_{j k}^{i} d x^{j}\right)\left(x_{t}, y_{t}\right)+\delta_{i}^{k}
$$

(see [16, p. 142]).

Whence

$$
A_{t}=\left[\begin{array}{cc}
0 & -\theta_{t} \\
\theta_{t} & 0
\end{array}\right]
$$

for $\theta_{t}=x_{t} / y_{t}$.

Thus $\left|A_{t}\right|=\sqrt{ } 2\left|\theta_{t}\right|$ (confirming the Killing form identity (12)), and

$$
\frac{1}{t} \log \left|T_{u} \xi_{t}(\omega)(v)\right|=\frac{1}{t} \log \left(1+2 \theta_{t}^{2}\right) \rightarrow 0
$$

by Lemma 5.6 , as required.

REMARK. Working in $U^{c}$ with $\left(x_{0}, y_{0}\right)=(0,1)$ it follows from (35) that the Type II orbit through $\left(x_{0}, y_{0}, L_{0}\right)$ is just the horizontal lift of the geodesic ray through $\left(x_{0}, y_{0}\right)$ in the direction $c$.

ACKNOWLEDGEMENTS. Our work on this project benefitted from contacts with P. Baxendale, M. Donsker, Y. Guivarc'h, A. Manning, M. Pinsky and L. C. G. Rogers. In particular L. C. G. Rogers taught us about the $h$-transform and $Y$. Guivarc' $h$ told us that the stable manifold structure for constant negative curvature should be as for the geodesics flow: the second author would like to thank him and IRMA Rennes for hospitality in September 1983. The work was helped by SERC grants GR/C/13644 and GR/C/60860. 


\section{REFERENCES}

1. V. I. Arnold and A. Avez, Ergodic problems of classical mechanics, Benjamin, New York, 1968.

2. P. H. Baxendale, Asymptotic behaviour of stochastic flows of diffeomorphisms: two case studies, Preprint, Dept. of Math., University of Aberdeen, Scotland, 1984.

3. A. P. Carverhill, Flows of stochastic dynamical systems: Ergodic theory, Stochastics 14 (1985), 273-318.

4. __ A formula for the Lyapunov numbers of a stochastic flow. Application to a perturbation theorem, Stochastics 14 (1985), 209-226.

5. ___ A "Markovian" approach to the multiplicative ergodic theorem for nonlinear stochastic dynamical systems, Preprint, Mathematics Institute, Univ. of Warwick, Coventry, England, 1984.

6. A. P. Carverhill and K. D. Elworthy, Flows of stochastic dynamical systems: the functional analytic approach, Z. Wahrsch. Verw. Gebiete 65 (1983), 245-267.

7. A. P. Carverhill, M. Chappell and K. D. Elworthy, Characteristic exponents for stochastic flows, Proc. Conf. 'BIBOS I: Stochastic Processes-Mathematics and Physics', Lecture Notes in Math., Springer-Verlag (to appear).

8. J. L. Doob, Classical potential theory and its probabilistic counterpart, Grundlehren Math. Wiss., vol. 262, Springer-Verlag, New York, Berlin, Heidelberg and Tokyo, 1984.

9. K. D. Elworthy, Stochastic dynamical systems and their flows, Stochastic Analysis (A. Friedman and M. Pinsky, eds.), Academic Press, London and New York, 1978, pp. 79-95.

10. __ Stochastic differential equations on manifolds, London Math. Soc., Lecture Notes in Math., Cambridge Univ. Press, 1982.

11. __ Stochastic dynamical systems and their Lyapunov exponents, Proceedings of VII ELAM, Simon Bolivar University, Caracus, July 1984, Lecture Notes in Math. (to appear).

12. A. Friedman, Non-attainability of a set by a diffusion process, Trans. Amer. Math. Soc. 197 (1974), 245-271.

13. Stochastic differential equations and applications, 2 volumes, Academic Press, London and New York, 1975.

14. N. Ikeda and S. Watanabe, Stochastic differential equations and diffusion processes, Kodansha, Tokyo, and North-Holland, Amsterdam, New York and Oxford, 1981.

15. Yu. Kifer, A multiplicative ergodic theorem for random transformations, Preprint, Institute of Mathematics, Hebrew University of Jerusalem, Jerusalem, 1984.

16. S. Kobayashi and K. Nomizu, Foundations of differential geometry, Vol. 1, Wiley-Interscience, New York and London, 1963.

17. ___, Foundations of differential geometry, Vol. II, Wiley-Interscience, New York, Chichester, Brisbane and Toronto, 1969.

18. M. P. Malliavin and P. Malliavin, Factorisations et lois limites de la diffusion horizontale au-dessus d'un espace Riemannien symmetrique, Theory du Potential et Analyse Harmonique (J. Farant, ed.), Lecture Notes in Math., vol. 404, Springer-Verlag, 1974.

19. ___ Holonomie stochastique au-dessus d'un espace riemannien symetrique, C. R. Acad. Sci. Paris Sér. A 280 (1975), 793-795.

20. P. Malliavin, Champs de Jacobi stochastiques, C. R. Acad. Sci. Paris Sér. A 285 (1977), 789-792.

21. V. I. Oseledec, A multiplicative ergodic theorem. Lyapunov characteristic exponents for dynamical systems, Trans. Moscow Math. Soc. 19 (1968), 197-231.

22. M. Pinsky, Stochastic Riemannian geometry, Probabilistic Analysis and Related Topics, vol. 1 (A. T. Bharucha Reid, ed.), Academic Press, London and New York, 1978.

23. J.-J. Prat, Etude asymptotique et convergence angulaire du mouvement brownien sur une variété à courbure négative, C. R. Acad. Sci. Paris Sér. A 280 (1975), 1539-1542.

24. M. S. Ragumathan, A proof of Oseledec's multiplicative ergodic theorem, Israel J. Math. 32 (1979), 356-362.

25. D. Ruelle, Ergodic theory of differentiable dynamical systems, Inst. Hautes Études Sci. Publ. Math., Bures-sur-Yvette, France, 1979.

26. D. Sullivan, The Dirichlet problem at infinity for a negatively curved manifold, J. Differential Geom. 18 (1983), 723-732. 
27. T. Sunada, Geodesic flows and geodesic random walks, Advanced Studies in Pure Math. 3 (1984), 47-85.

28. K. Yosida, Functional analysis, 2nd ed., Grundlehren Math. Wiss., vol. 123, SpringerVerlag, Berlin, Heidelberg and New York, 1968.

MATHEMATICS INSTITUTE, UNIVERSITY OF WARWICK, COVENTRY CV4 7AL, ENGLAND (Current address of K. D. Elworthy)

Current address (A. P. Carverhill): Operational Research Group, Bank of England, Threadneedle Street, London EC2, England 